\title{
Design and Operation of a Multiple-Cathode, High-Power, Rectangular Discharge Chamber
}

\author{
Joshua L. Rovey ${ }^{*}$ and Alec D. Gallimore ${ }^{\dagger}$ \\ Plasmadynamics and Electric Propulsion Laboratory \\ Department of Aerospace Engineering \\ University of Michigan \\ 1919 Green Rd. Rm. B107 \\ Ann Arbor, MI 48109 USA \\ Phone: 734-764-4199 \\ Fax: 734-763-7158
}

\begin{abstract}
A high-power, rectangular discharge chamber is being designed by the University of Michigan for operation with multiple discharge cathode assemblies (DCAs). The multiple cathode approach attempts to increase thruster lifetime by operating three DCAs sequentially, possibly providing a threefold increase in discharge life. The baseline multiplecathode discharge chamber (MCDC) magnetic field topology is developed based on the NASA Evolutionary Xenon Thruster (NEXT) magnetic field. The selected MCDC magnetic field consists of permanent magnet rings, an electromagnet, and magnetic iron c-channels to augment the field. Experimental results are obtained by operating the MCDC with an ion collection grid (without beam extraction) in the University of Michigan Large Vacuum Test Facility. Operation of the MCDC with the active DCA located on centerline and offcenterline is accomplished, as well as operation with the dormant cathodes floating and connected to cathode common. Different magnetic field configurations are experimentally tested by adjusting the electromagnet current or adding the iron c-channels. Discharge stability is analyzed by measuring discharge voltage oscillations, and 13 button probes are placed on the ion collection grid to determine uniformity. MCDC grid-plane plasma properties, backplate electron current, and dormant cathode current and voltage characteristics are also monitored. A stable discharge is obtained for all operational configurations. Results indicate that the $0 \mathrm{~A}$ electromagnet configuration provides the best performance and flatness with optimum values of $194 \mathrm{~W} / \mathrm{A}$ at 0.89 propellant efficiency and 0.55, respectively. Backplate electron current ratios indicate that the majority of the discharge current is deposited in the corners of the rectangular MCDC. Finally, operation of the dormant cathodes with propellant flow is suggested to reduce potential erosion of those units.
\end{abstract}

$\mathrm{A}_{\mathrm{p}} \quad$ Langmuir probe area $\left(\mathrm{mm}^{2}\right)$

$\mathrm{I}_{1}, \mathrm{I}_{2} \quad$ Langmuir probe current values (A)

$\mathrm{I}_{\mathrm{emag}} \quad$ Electromagnet current (A)

$\mathrm{I}_{\mathrm{se}} \quad$ Electron saturation current (A)

$\mathrm{I}_{\mathrm{si}} \quad$ Ion saturation current (A)

$\mathrm{J}_{\mathrm{b}} \quad$ Beam current (A) (Current leaving MCDC)

$\mathrm{J}_{\mathrm{d}} \quad$ Discharge current (A)

$\mathrm{J}_{\mathrm{g}} \quad$ Grid Plane ion current $(\mathrm{A})\left(\mathrm{J}_{\mathrm{b}}+\mathrm{J}_{\mathrm{s}}\right)$

$\mathrm{J}_{\mathrm{S}} \quad$ Ion collection grid current (A)

$\mathrm{k}_{\mathrm{B}} \quad$ Boltzmann's constant $\left(1.38 \times 10^{-23} \mathrm{~J} / \mathrm{K}\right)$

$\hat{\dot{m}} \quad$ Reduced mass flow rate (equivalent Amps)

\section{Nomenclature}

$\mathrm{M}_{\mathrm{i}} \quad$ Xenon ion mass $\left(2.18 \times 10^{-25} \mathrm{~kg}\right)$

$\mathrm{m}_{\mathrm{e}} \quad$ Electron mass $\left(9.11 \times 10^{-31} \mathrm{~kg}\right)$

$\mathrm{n}_{\mathrm{e}} \quad$ Electron number density $\left(\mathrm{cm}^{-3}\right)$

$\mathrm{n}_{\mathrm{i}} \quad$ Ion number density $\left(\mathrm{cm}^{-3}\right)$

$\mathrm{P}_{\mathrm{b}} \quad$ Base pressure (Torr)

$\mathrm{P}_{\mathrm{c}} \quad$ Corrected pressure (Torr)

$\mathrm{P}_{\mathrm{i}} \quad$ Indicated pressure (Torr)

$\mathrm{r} \quad$ Langmuir probe radius $(\mathrm{mm})$

$\mathrm{T}_{\mathrm{e}} \quad$ Electron temperature $(\mathrm{eV})$

$\mathrm{V}_{1}, \mathrm{~V}_{2}$ Langmuir probe voltage values $(\mathrm{V})$

$\mathrm{V}_{\mathrm{d}} \quad$ Discharge Voltage $(\mathrm{V})$

\footnotetext{
${ }^{*}$ Graduate Student, Aerospace Engineering, AIAA Student Member

${ }^{\dagger}$ Professor, Aerospace Engineering, AIAA Associate Fellow

Copyright 2005 by Joshua L. Rovey

Published by the American Institute of Aeronautics and Astronautics, Inc. with permission.
} 


$$
\begin{array}{ll}
\eta_{u d} & \text { Propellant utilization efficiency (\%) } \\
\varepsilon_{b} & \text { Ion production cost (W/A) } \\
\lambda_{D} & \text { Debye length }(\mathrm{mm})
\end{array}
$$

$\phi_{i} \quad$ High-voltage optics ion transparency $(\sim 70 \%)$

$\hat{\phi}_{i} \quad$ Ion collection grid ion transparency

\section{Introduction}

$\mathrm{N}_{\mathrm{p} \text { ivis }}^{\mathrm{A}}$ ASA's Project Prometheus is advancing the future of space exploration by developing nuclear electric propulsion (NEP) technology for deep space missions. Ion thrusters are high-efficiency, high-specific impulse propulsion systems that are being proposed as the primary propulsion source for such missions. An ion thruster that can satisfy the mission requirements will require long life, high-power, and high-specific impulse. NASA Glenn Research Center (GRC) has developed such an ion thruster through the High Power Electric Propulsion (HiPEP) project, ${ }^{1-3}$ and is currently focused on designing the Herakles ion thruster with the NASA Jet Propulsion Laboratory (JPL). Because an ion thruster must operate continuously for perhaps as long as 7-14 years for Prometheus-class missions, ${ }^{4-6}$ assessing and increasing thruster lifetime is of foremost importance.

The potential failure mechanisms for ion thrusters are generally classified into four categories: 1.) discharge cathode failure, 2.) neutralizer failure, 3.) ion optics failure, and 4.) electron backstreaming. Erosion of the screen and accelerator grids due to ion impingement is the primary cause of failure mode 3. As the accelerator grid apertures widen due to erosion, mode 4 becomes important because the number of backstreaming electrons increases and eventually destroys the cathode. Methods for increasing accelerator grid lifetime and reducing electron backstreaming have been developed. ${ }^{7-10}$ Failure of the hollow cathode is the primary cause of modes 1 and 2 . Hollow cathode failure is known to be caused by either depletion of the barium insert, the formation of tungstates in the barium, or physical erosion. Consequently, the lifetime of the NASA Solar Electric Propulsion Technology and Applications Readiness (NSTAR) ion thruster is limited to $\sim 30,000$ hours ${ }^{11,12}$ and recent results suggest the NASA Evolutionary Xenon Thruster (NEXT) may have a comparable lifetime. ${ }^{13,14}$ It is important to note that these thruster lifetimes are insufficient for missions requiring continuous operation for greater than approximately 3-5 years.

In order to increase thruster lifetime for Prometheus-class missions, the University of Michigan Plasmadynamics and Electric Propulsion Laboratory (PEPL) is developing an ion thruster that utilizes three sequentially operated discharge cathodes. With this approach, a new discharge cathode is ignited when the previous one fails. Ideally this will increase thruster discharge lifetime threefold, making longer mission times a possibility. The following sections describe the design of the multiple-cathode discharge chamber (MCDC), apparatus used to operate the MCDC as a simulated ion thruster, ${ }^{15}$ results, analyses, and conclusions.

\section{Multiple-Cathode Discharge Chamber (MCDC)}

In an effort to extend the lifetime of an ion thruster, the University of Michigan PEPL is developing a HiPEPderivative ion thruster that operates three discharge cathode assemblies (DCAs) sequentially. Investigation of various MCDC designs is initiated using the GRC HiPEP engine baseline dimensions and the NEXT magnetic field topology. Several magnetic field designs are investigated and "graded" based on a set list of criteria. Each design is solved numerically using the 3D magnetostatic code $\mathrm{MagNet}^{\mathrm{TM}} 6.0$, which provides a magnetic field topology from which each design is evaluated. The following sections describe the criteria used to determine the selected discharge chamber magnetic field, the designs that are numerically investigated, the results of the numerical solutions, and the PEPL MCDC.

\section{A. Design Criteria}

In order to facilitate the operation of three DCAs, a discharge chamber must have a magnetic field that fulfills certain criteria. More specifically, the magnetic field must exhibit similar characteristics at each of the three DCA locations inside the chamber. The PEPL design goal is to place each DCA in a magnetic field environment similar to the NEXT DCA. Accomplishing this objective assures that each DCA will function in a manner similar to the proven electron source of the NEXT ion engine. The selected MCDC design is required to exhibit the following characteristics. First, the magnetic field lines at the exit of the cathodes are uniform and parallel to the cathode's axis. This allows exiting electrons to essentially spiral away along the field lines. ${ }^{16,17}$ Second, the exit of the cathode is located downstream of the peak magnetic field strength. This feature assists electrons in falling away from the cathode and out into the discharge chamber. Placing the cathode upstream of the peak field strength may lead to a mirroring effect. ${ }^{16,17}$ Third, the magnitude of the flux at the cathode exit plane is equal to the NEXT field strength in order to facilitate the collision processes necessary for propellant ionization. Finally, the permanent magnet ring spacing is designed such that the intercusp field strength is similar to NEXT. 


\section{B. Discharge Chamber Models}

In order to evaluate each MCDC design, the 3D magnetostatic code $\mathrm{MagNet}^{\mathrm{TM}} 6.0$ is used to numerically solve for the magnetic field topology produced by each of the designs. Each design is modeled and solved in order to evaluate its ability to produce a NEXT-like magnetic field at each of the three DCA locations and throughout the discharge chamber. Many different MCDC designs are modeled and each differs primarily by the number, spacing, and shape of the permanent magnet rings. Each design consists of a rectangular, non-magnetic stainless steel anode shell with samarium cobalt permanent magnet rings located on both the anode walls and backplate. Some of the variables manipulated between models are listed below.

- Number, Shape, Location of Backplate Magnet Rings -Shapes: All Rectangular, Combination Circular and Rectangular, Hexagonal -Number: 2, 3, 4, 5, or 6 total rings

- Number, Location of Anode Wall Magnet Rings -Number: 2, 3, or 4 total rings

- Permanent magnet size (width $1.27 \mathrm{~cm}$ or $0.64 \mathrm{~cm}$ ) (thickness $0.51 \mathrm{~cm}$ or $1.02 \mathrm{~cm}$ )

- DCA locations on Backplate

\section{Modeling Results}

Using the solved magnetic field topology provided by $\mathrm{MagNet}^{\mathrm{TM}}$ 6.0, each design is analyzed and evaluated on its ability to create a NEXT-like magnetic field environment at each of the three DCA locations and throughout the discharge chamber. Many of the designs are quickly eliminated due to violation of the criteria outlined above. Specifically, those designs that do not place the DCAs inside a ring of permanent magnets are rejected because the direction of the magnetic field lines at the cathode exit plane inhibit electrons from escaping the cathode and entering the discharge chamber. Utilizing the smaller magnet dimensions to produce the desired magnetic field requires more total magnet rings and subsequently more magnetic cusps. Because the majority of plasma electrons are collected at the cusps, ${ }^{18-22}$ decreasing the total rings can lead to increased electron collection at intercusp surfaces. ${ }^{23}$ However, NEXT-like intercusp field strengths can still be obtained using fewer rings and the larger magnets. Additionally, utilizing fewer rings reduces the overall mass of the engine.

\section{Selected MCDC}

\section{Anode}

The selected MCDC has a rectangular shape and a rectangular ion extraction area of $3600 \mathrm{~cm}^{2}$. Non-magnetic stainless steel sheet metal is used to construct the backplate and the rectangular shell. Four corner brackets are welded to the sheet metal to form the rectangular shell and the backplate is bolted to the shell to form the five sided rectangular anode. Three holes centered in the backplate are spaced a few centimeters linearly apart for placement of the DCAs.

\section{Permanent Magnets}

Samarium Cobalt $\left(\mathrm{Sm}_{2} \mathrm{Co}_{17}\right)$ permanent magnets are utilized to form the baseline magnetic circuit. Three circular magnet rings are formed and mounted to the backplate at each DCA location. Two concentric rectangular rings surround the three DCAs and two more rectangular rings are located on the anode walls. Simulations show that this configuration places each DCA in a NEXT-like magnetic field environment and provides similar intercusp field strengths.

\section{Electromagnet}

In addition to the baseline all-permanent-magnet magnetic circuit, an electromagnet is utilized to augment and change the magnetic field configuration. A coil with 280 turns of 15 -gauge magnetic wire is wrapped in a doubleconductor configuration around a rectangular aluminum bobbin and placed inside a magnetic iron channel. The use of two conductors reduces the length of the wire and subsequently its resistance so that a lower voltage can be used to drive the electromagnet current. The magnetic channel increases the efficiency of the electromagnet by directing the flux, thus the electromagnet requires fewer turns and less current than if operated without the magnetic iron. The near-DCA magnetic field is adjusted by mounting the electromagnet externally to the backplate and supplying a current within the range of \pm 10 A. Negative current decreases the near-DCAs magnetic field and positive current 
increases it. With the electromagnet attached, the DCA exit plane magnetic field strength can be adjusted from $15 \mathrm{G}$ to over $100 \mathrm{G}$. In order to recover the baseline all-permanent-magnet configuration, the electromagnet must be operated at $-5 \mathrm{~A}$.

\section{Magnetic Field Configurations}

Although an infinite number of magnetic field configurations are possible because the electromagnet can be set at any desired current level, only seven are investigated in this work. Five magnetic field configurations are studied by setting the electromagnet current at $\pm 10 \mathrm{~A}, \pm 5 \mathrm{~A}$, and $0 \mathrm{~A}$. A sixth configuration encloses the $50 \mathrm{G}$ line within the MCDC by attaching the electromagnet (operated at $0 \mathrm{~A}$ ) and 4 magnetic iron c-channels to each of the anode walls. Placing the magnetic iron c-channels at the cusp locations increases the strength of the intercusp field and effectively encloses the $50 \mathrm{G}$ line. By definition the $50 \mathrm{G}$ line is considered enclosed if it does not intersect with the anode walls or backplate. The seventh configuration is asymmetric with an increase in magnetic field strength near the offcenterline DCA. This configuration may force the peak current density location to shift from directly downstream of the off-centerline DCA.

\section{E. Comparison of Experimental and Simulated Magnetic Fields}

In order to validate the designed MCDC magnetic field topology, 2-D experimental magnetic field maps are compared with the simulation data over 6 planes for 6 magnetic field configurations. Excellent agreement is obtained between the experimentally measured profiles and the $\mathrm{MagNet}^{\mathrm{TM}}$ simulations. At spatial locations near the permanent magnets an average percent difference of approximately $\pm 15 \%$ is obtained. Furthermore, the experimental maps verify that each DCA is located in a similar magnetic field environment. Comparisons of the allpermanent-magnet and 5 electromagnet configurations show that a $-5 \mathrm{~A}$ electromagnet current is required to recover the all-permanent-magnet case. This result also suggests that it may be possible to recover any all-permanentmagnet magnetic field from the electromagnet augmented configuration. Finally, verification of the enclosure of the $50 \mathrm{G}$ line is obtained. This configuration requires the electromagnet and the magnetic iron c-channels to effectively keep the $50 \mathrm{G}$ contour line from intersecting the anode.

\section{Experimental Apparatus and Setup}

\section{A. Vacuum Facility}

The University of Michigan Large Vacuum Test Facility (LVTF) is used for all experiments presented here. The LVTF is a stainless-steel vacuum chamber with a diameter of $6 \mathrm{~m}$ and a length of $9 \mathrm{~m}$. Four $400 \mathrm{CFM}$ mechanical pumps and two 2,000 CFM blowers evacuate the chamber to a moderate vacuum ( 30 - 100 Torr). In order to reach high vacuum, the facility employs seven CVI TM-1200 re-entrant cryopumps, each of which is surrounded by an $\mathrm{LN}_{2}$ baffle. The cryopump system can be operated with any number of pumps in use. With all seven pumps operating, the facility pumping speed is $240,000 \mathrm{l} / \mathrm{s}$ on xenon with a base pressure of $<2.0 \times 10^{-7}$ Torr. For the experiments described here only two cryopumps are operated, which yields a base pressure of $5.2 \times 10^{-7}$ Torr. The chamber pressure is monitored using two hot-cathode ionization gauges. The first gauge, a Varian model 571 gauge with an HPS model 919 Hot Cathode Controller, is connected to the chamber by a 25 -cm-long by 3.48 -cm-innerdiameter tube. The second is a Varian model UHV-24 nude gauge with a Varian UHV senTorr Vacuum Gauge Controller. Pressure measurements from the gauges are corrected for xenon using the known base pressure on air and a correction factor of 2.87 for xenon according to the following equation ${ }^{24}$

$$
P_{c}=\frac{P_{i}-P_{b}}{2.87}+P_{b}
$$

where $\mathrm{P}_{\mathrm{c}}$ is the corrected pressure on xenon, $\mathrm{P}_{\mathrm{b}}$ is the base pressure, and $\mathrm{P}_{\mathrm{i}}$ is the indicated pressure when xenon is flowing into the vacuum chamber. Corrected pressure for the nude ion gauge is reported as the background pressure in the chamber. A recent investigation of the pressure inside the LVTF during Hall thruster cold-flow operation has shown that the nude gauge provides better agreement with the true pressure of the facility. ${ }^{25}$ Corrected operating pressures for all experiments reported here are below $4.0 \times 10^{-6}$ Torr on xenon. 


\section{B. MCDC Test Article (TA)}

\section{Hardware Setup}

The MCDC described in Section II is placed inside the LVTF and the electromagnet is mounted to the backplate for all experiments. Those experiments requiring enclosure of the $50 \mathrm{G}$ line also utilize the magnetic iron c-channels mounted to the anode walls. Two dormant cathode units (DCUs) are mounted at two of the DCA locations. Each DCU is designed and constructed to appear as similar to the active DCA as
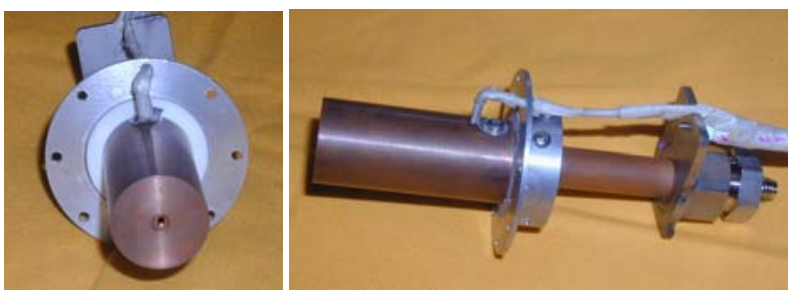

\author{
Figure 1: Photographs of the Dormant Cathode \\ Units (DCUs).
} possible. Each DCU has a copper cathode tube with a chamfered orifice and a surrounding copper keeper tube with an orifice. Copper is utilized due to its relatively large sputtering yield ${ }^{26,27}$ in order to visualize any erosion phenomena that may be present during TA operation. Hightemperature ceramic (Macor) is used to hold the cathode and keeper tubes at the required orifice spacing. An aluminum mounting flange attaches to the Macor. Along with the active DCA, each DCU is also connected to a propellant feed system through a propellant isolator. This allows the effect of propellant flow through the DCUs on TA performance, grid-plane uniformity, and DCU erosion to be studied. Photographs of the DCUs are shown in Figure 1.

Because only discharge chamber performance is being investigated, the TA is operated as a simulated ion thruster without beam extraction. ${ }^{15}$ Simulated operation is accomplished by mounting an ion collection grid at the ion extraction plane, high-voltage ion optics are not required. The ion collection grid is constructed of non-magnetic stainless steel with holes arranged in a staggered pattern over the active area of the grid. The open area fraction of the ion collection grid is measured to be similar to the HiPEP ion optics open area fraction. ${ }^{28}$

A reverse-feed plenum is designed based on the NEXT propellant feed system. This type of configuration has been shown to increase ion engine performance. ${ }^{29}$ A NEXT hollow cathode with a surrounding keeper is utilized in the TA. Only one DCA is used for all experiments presented here. The DCA is simply moved from the center to the left position for off-centerline, left DCA operation. Attachment of the ion collection grid, the NEXT DCA, the DCUs, and the plenum to the MCDC is referred to as the MCDC test article (TA) or simply, TA.

\section{Electrical Setup}

Electrically the TA is setup for simulated ion thruster operation described by Brophy. ${ }^{15}$ An engine bias supply is used to raise the TA cathode potential above facility ground. This prevents electrons from leaving the TA and also allows the extracted current to be measured. The collection grid is biased negative of cathode potential to measure the ion current to the grid. Each DCA or DCU is electrically connected to or isolated from the circuit by two switches: one for the cathode and one for the keeper. During TA operation the DCA is always connected to the circuit and the DCUs are connected or isolated from the circuit by using the switches. Each of the three keepers is connected to the anode through a $10 \mathrm{k} \Omega$ resistor. The electromagnet is electrically isolated from the TA.

\section{Diagnostics}

Two types of diagnostics are utilized to obtain information about the plasma discharge produced by the TA. Button probes are designed and used to obtain ion current density and electron current density measurements at specific locations on the TA. A Langmuir probe is mounted to the ion collection grid to obtain grid-plane plasma property data.

\section{Button Probes}

In order to obtain an approximation of the grid-plane current density distribution, 13 button probes are placed at multiple locations on the ion collection grid. Intercusp electron collection on the anode backplate is monitored with 2 button probes, one at the corner and one at the mid-plane of the backplate intercusp region. Each button probe consists of a $0.32-\mathrm{cm}$-diameter stainless steel rod flush mounted inside an alumina tube. The button probes are essentially planar Langmuir probes except the bias voltage is no longer adjusted but held constant in either the ion saturation (ion current) or electron saturation (electron current) regime. Collected current is calculated by measuring the voltage drop across a current shunt and dividing by the known resistance $(10 \mathrm{k} \Omega)$. A schematic of the probes and their electrical setup is shown in Figure 2. 
Because the magnetic circuit of the MCDC utilizes rectangular magnet rings, the electron deposition to the TA corners is characterized. Two button probes are mounted to the backplate of the anode on the left side of the left DCA and biased to collect electron current. Both probes are located midway between the magnetic cusps; i.e., in the middle of the intercusp region. Probe number 1 is placed in the corner of the rectangular TA, while number 2 is placed at the mid-plane. This allows the intercusp corner electron deposition to be compared with that obtained at the mid-plane. The results presented here utilize the ratio of the currents (corner probe current, probe 1, divided by mid-plane probe current, probe 2) Probe locations on the MCDC TA are shown in Figure 3.

\section{Single Langmuir Probe}

Along with the button probes, a single Langmuir probe is also placed on the ion collection grid to determine plasma properties at the grid plane. The electrode of the probe is sized such that the probe operates in the thin sheath regime. At the grid plane, the number density and electron temperature are expected to have values within the ranges of $10^{10}-10^{11} \mathrm{~cm}^{-3}$ and $2-13 \mathrm{eV},{ }^{30-34}$ respectively. The relationship of the Debye length to electron number density and temperature is illustrated in the following equation. . $6,17,35^{2}$

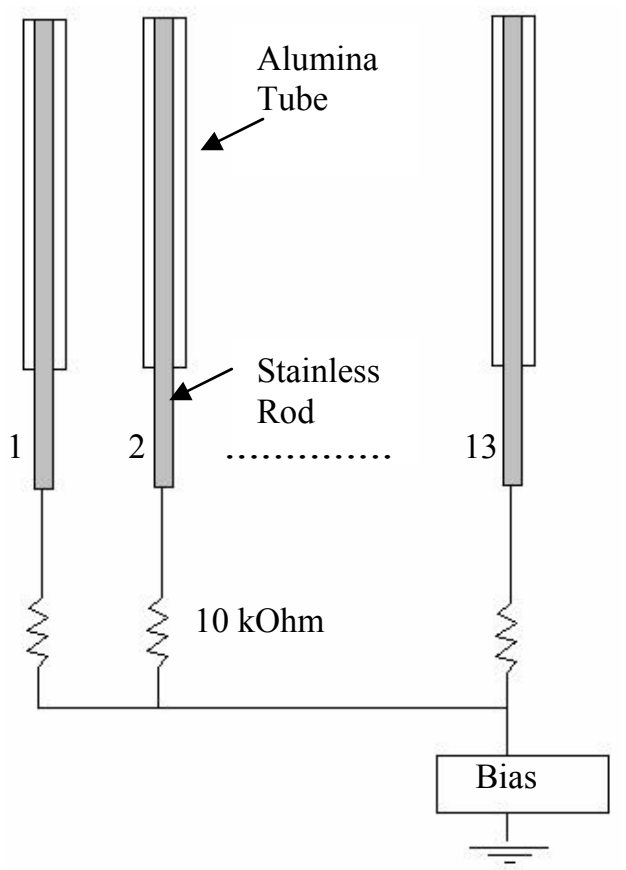

Figure 2: Schematic of button probes and electrical setup.

$$
\lambda_{D}=743 \sqrt{\frac{T_{e}[\mathrm{eV}]}{n_{e}\left[\mathrm{~cm}^{-3}\right]}}
$$

In the thin sheath regime, the flux of particles entering the sheath can be calculated without considering the details of the orbits of these particles in the sheath. ${ }^{36-38}$ For a large ratio of probe radius, $r$, to Debye length, $\lambda_{D}$, the collection area of the probe can be approximated as the area of the probe. ${ }^{36-38} \mathrm{~A}$ single Langmuir probe with a 5.08$\mathrm{mm}$-diameter tungsten electrode housed inside two concentric alumina tubes with outer diameters of $6.60 \mathrm{~cm}$ and $1.63 \mathrm{~cm}$ is used for all experiments. A large length to diameter ratio is utilized to minimize end effects and the total probe area is calculated to be $8.821 \mathrm{~mm}^{2}$. The probe is mounted to the grid and the electrode extends approximately $3 \mathrm{~cm}$ into the discharge chamber.

A Hiden Analytical system utilizing ESPsoft software obtains the I-V characteristics. However, the results calculated by the Hiden system assume the probe is operated in the Orbital Motion Limited (OML) regime, so a thin sheath analysis is manually applied to the I-V characteristics to determine the floating potential, plasma potential, electron temperature, electron number density, and ion number densities. Floating potential is defined as the potential at which the probe collects zero current and plasma potential is calculated by finding the maximum in the derivative of the I-V curve. The following equations are used in the analysis.

$$
\begin{aligned}
T_{e}[e V] & =\frac{\left(V_{1}-V_{2}\right)}{\ln \left(I_{1} / I_{2}\right)} \\
I_{s e} & =e n_{e} \sqrt{\frac{k_{B} T_{e}}{2 \pi m_{e}}} A_{p} \\
I_{s i} & =0.61 e n_{i} \sqrt{\frac{k_{B} T_{e}}{M_{i}}} A_{p}
\end{aligned}
$$


In these equations, $T_{e}$ is electron temperature, $V_{1}$ and $V_{2}$ are probe voltages in the electron retarding regime, $I_{1}$ and $I_{2}$ are probe currents in the retarding regime, $\mathrm{I}_{\mathrm{se}}$ is electron saturation current, e is the elementary charge $\left(1.6 \times 10^{-19} \mathrm{C}\right)$, $\mathrm{n}_{\mathrm{e}}$ is electron number density, $\mathrm{k}_{\mathrm{B}}$ is Boltzmann's constant $\left(1.38 \times 10^{-23} \mathrm{~J} / \mathrm{K}\right), \mathrm{m}_{\mathrm{e}}$ is the mass of an electron $\left(9.11 \times 10^{-31}\right.$ $\mathrm{kg}), \mathrm{A}_{\mathrm{p}}$ is the probe area $\left(8.821 \mathrm{~mm}^{2}\right), I_{\mathrm{si}}$ is the ion saturation current, $n_{i}$ is ion number density, and $\mathrm{M}_{\mathrm{i}}$ is the ion mass $\left(\mathrm{M}_{\mathrm{Xe}}=2.18 \times 10^{-25} \mathrm{~kg}\right)$.

\section{Data Acquisition Systems}

TA operating parameters and the 15 button probe currents (13 grid-plane ion current and 2 backplate electron current probes) are recorded using a 34970A 22-bit Agilent data logger with 2 - 34901A 20-channel multiplexers (a total of 40 channels are available). Depending on the electrical setup, the data logger records discharge current and voltage, three cathode and keeper currents, three cathode and keeper floating voltages, collection grid bias voltage and current, cathode common bias voltage and extracted current, cathode heater voltage and current, electromagnet voltage and current, and the 15 button probe currents. In the case where a current is recorded, the voltage across a current shunt is measured and then divided by the known resistance to determine the current. With this setup realtime performance data are obtained and data collection is extremely time efficient. One sweep through all utilized channels requires approximately 1.5 seconds.

A Tektronix TDS 3034B oscilloscope is utilized to measure discharge voltage oscillations. Reported peak-topeak voltage oscillations are the difference between maximum and minimum values recorded during a 4 msec oscilloscope trace sweep. Typical voltage oscillations for operating conditions reported here have peak-to-peak oscillations less than $1.5 \mathrm{~V}$.

\section{Initial TA Operation}

Initially a coordinate system is constructed such that the center DCA opening in the backplate of the TA is considered the origin. Looking downstream from behind the TA, the positive Z-axis extends in the downstream direction, the positive $\mathrm{X}$-axis extends to the left, and the positive $\mathrm{Y}$-axis extends in the upward direction. A graphical representation of the coordinate system is shown in Figure 3.

DCA and main plenum beam-extraction flow rates must be reduced for simulated ion thruster operation in order to maintain the thruster internal neutral density. A relationship for the reduced flow rate based on the beam-extraction flow rate has been determined by Brophy. ${ }^{15}$ However, because the TA has never been operated with beam-extraction a reduced flow rate cannot be calculated. The GRC HiPEP beam-extraction flow rate $^{3}$ cannot be used because the TA has an entirely different magnetic field configuration. The TA reduced flow rate for simulated ion thruster operation must be determined experimentally by monitoring the grid-plane ion current as a function of flow rate.

\section{A. Flow Rate Determination}

The grid-plane ion current is monitored while both the DCA and main plenum flow rates are adjusted. The flow rate corresponding with the maximum grid-plane ion current is assumed to be the reduced flow rate. These experiments are conducted for both centerline and off-centerline DCA positions and for multiple magnetic field configurations. A wait time of approximately 2 minutes is used to allow the flow rate adjustment to equilibrate inside the TA.

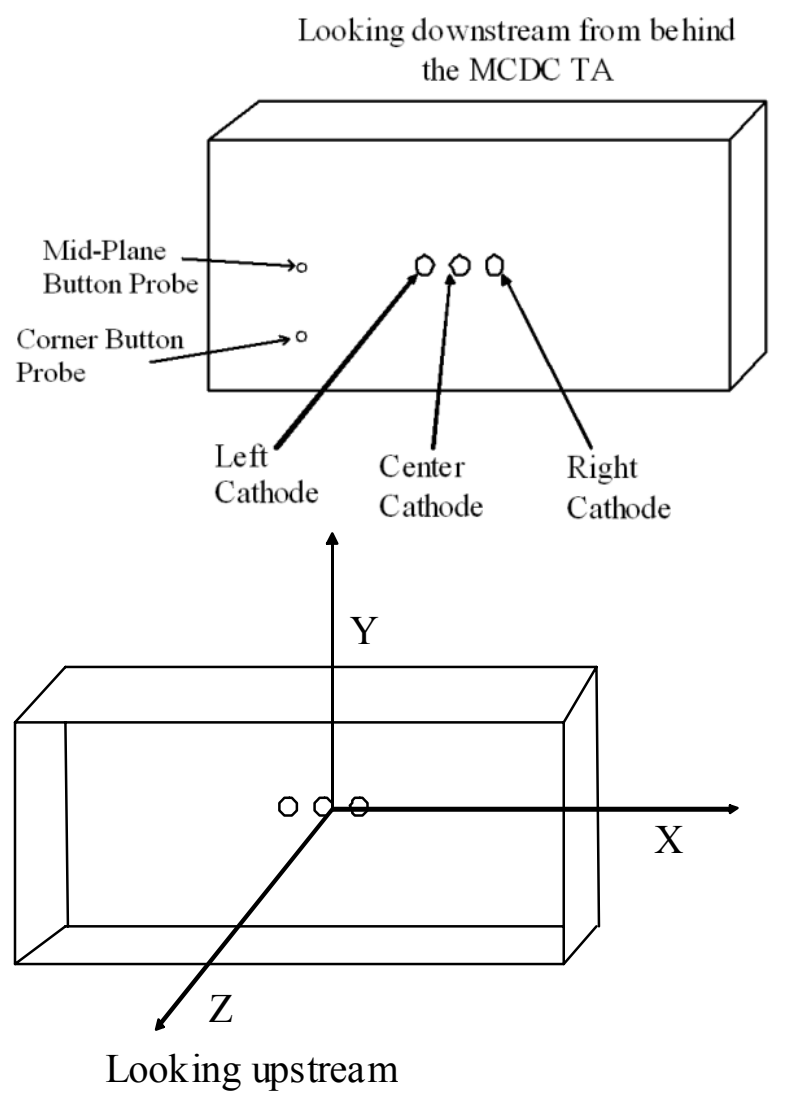

Figure 3: Coordinate system for the TA. 

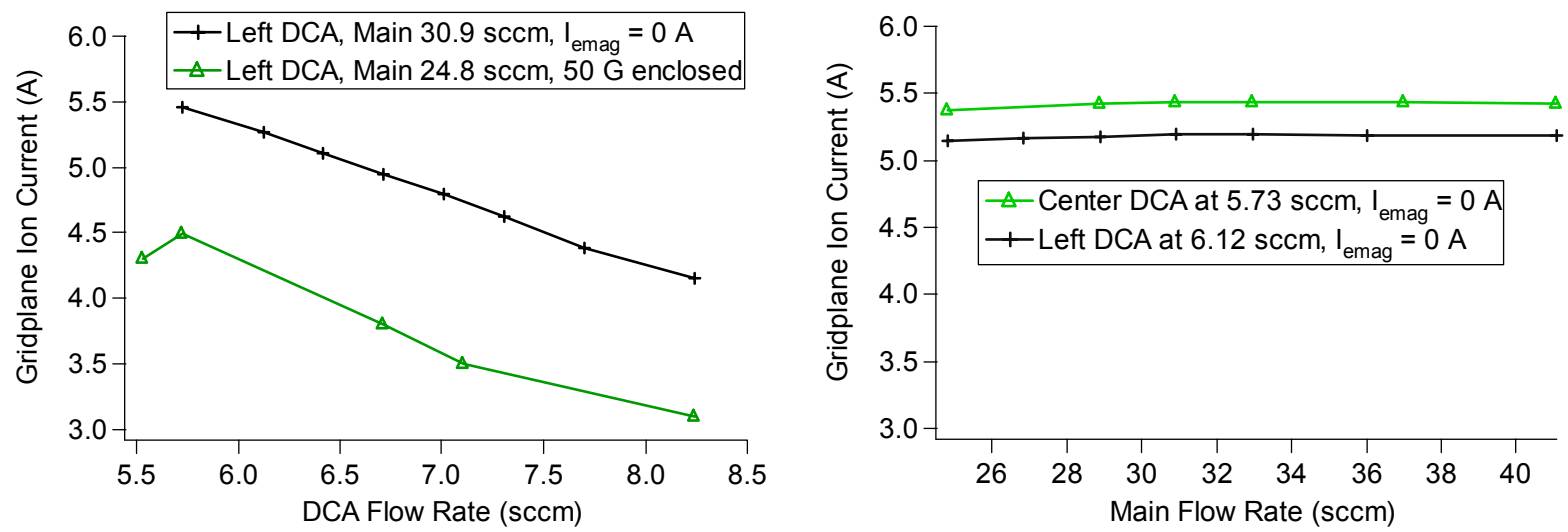

Figure 4: Flow rate study for determining DCA and main reduced flow rates.

Initially the DCA and main plenum flow rates are set at the GRC HiPEP beam extraction level. ${ }^{3}$ First the main plenum flow is held constant while the DCA flow is reduced. As the DCA flow decreases the grid-plane current increases and peak-to-peak discharge voltage oscillations increase for all DCA and magnetic field configurations investigated. The DCA flow rate that provides the largest grid-plane current and relatively low peak-to-peak discharge voltage oscillations is considered the optimized, reduced DCA flow rate. In some cases the DCA flow is optimized at $5.73 \mathrm{sccm}$. However, for other cases the flow is set to $6.12 \mathrm{sccm}$ because lower flow rates cause peak-to-peak discharge voltage oscillations greater than $5 \mathrm{~V}$. Large voltage oscillations occur when the cathode operates in "plume mode", which can be detrimental to cathode life. ${ }^{39}$ Previous researchers have suggested that peak-to-peak voltage oscillations should be

\begin{tabular}{|l|l|c|c|}
\hline DCA Activity & $\begin{array}{c}\text { Magnetic Field } \\
\text { Configuration }\end{array}$ & $\begin{array}{c}\text { DCA } \\
\text { (sccm) }\end{array}$ & $\begin{array}{c}\text { Main } \\
\text { (sccm) }\end{array}$ \\
\hline \multirow{5}{*}{ Left Active } & 50 G Enclosed & 5.73 & 24.8 \\
& Asymmetric & 6.12 & 30.9 \\
& Electromagnet -10 A & 6.12 & 30.9 \\
& Electromagnet -5 A & N/A & N/A \\
& Electromagnet 0 A & 6.12 & 30.9 \\
& Electromagnet +5 A & 6.12 & 30.9 \\
& Electromagnet +10 A & 6.12 & 30.9 \\
\hline & 50 G Enclosed & 5.73 & 24.8 \\
& Electromagnet -10 A & 5.73 & 30.9 \\
Center Active & Electromagnet -5 A & N/A & N/A \\
& Electromagnet 0 A & 5.73 & 30.9 \\
& Electromagnet +5 A & 5.73 & 30.9 \\
& Electromagnet +10 A & 5.73 & 30.9 \\
\hline
\end{tabular}

Table 1: Results of the reduced flow rate study for all DCA and magnetic field configurations investigated. less than $5 \mathrm{~V}$ during the preferred "spot mode" operation. ${ }^{11,12}$

After determining the DCA flow rate, the main plenum flow rate is obtained by setting the DCA flow to its reduced value and then decreasing the main plenum flow. As the plenum flow decreases an increase in peak-to-peak discharge voltage oscillations is observed for all DCA and magnetic field configurations. A peak in the grid-plane current is typically obtained at a main plenum flow rate of $30.9 \mathrm{sccm}$. However, for the $50 \mathrm{G}$ enclosed magnetic field configuration, the peak occurs at $24.8 \mathrm{sccm}$. An example of the trends seen during this study is shown in Figure 4.

Based on these results the DCA and main plenum flow rates for all investigated DCA and magnetic field configurations are determined and these results are summarized in Table 1. For the configuration with the electromagnet at $-5 \mathrm{~A}$, the peak-to-peak discharge voltage oscillations increase significantly above $5 \mathrm{~V}$ and therefore data are not collected at this condition. More information regarding this configuration is provided later in this paper.

\section{B. Bias Voltage Studies}

In conjunction with the flow rate study, bias voltage studies are conducted to ensure that the ion collection grid, cathode common bias, and button probes are in the saturation regime. Bias voltage studies are completed by adjusting the bias voltage and monitoring the collected current. For all results reported here, cathode common is biased $+25 \mathrm{~V}$ above facility ground, the ion collection grid and grid-plane button probes are biased $-20 \mathrm{~V}$ with respect to cathode common, and the backplate button probes are biased $+35 \mathrm{~V}$ with respect to cathode common. 


\section{Performance and Flatness Calculations}

Analysis of the performance and beam flatness of the TA is critical for determining its ability to function as an efficient ion thruster discharge chamber. Performance curves are usually generated by plotting ion production costs as a function of propellant utilization efficiency., ${ }^{3,15,34}$ The following equations are utilized to determine the performance of the TA, ${ }^{15}$

$$
\begin{gathered}
\hat{\phi}_{i}=\frac{0.35 J_{s}+J_{b}}{J_{g}} \\
\eta_{u d}=\frac{\phi_{i} J_{g}}{\hat{\dot{m}}+\phi_{i} J_{g}\left(1-\frac{\hat{\phi}_{i}}{\phi_{i}}\right)} \\
\varepsilon_{b}=\frac{\left(J_{d}-\phi_{i} J_{g}\right) V_{d}}{\phi_{i} J_{g}}
\end{gathered}
$$

where $J_{g}$ is the grid-plane ion current (summation of $J_{s}$, current collected by the grid, and $J_{b}$, the extracted or beam current), $\phi_{i}$ is the high-voltage ion optics transparency to ions (estimated at $70 \%$ ), ${ }^{28} \eta_{u d}$ is the propellant utilization efficiency, $\hat{\dot{m}}$ is the reduced propellant flow rate in equivalent amperes, $\hat{\phi}_{i}$ is the ion collection grid transparency to ions, $J_{d}$ is the discharge current, $V_{d}$ is the discharge voltage, and $\varepsilon_{b}$ is the ion production cost in W/A. The parameters $\varepsilon_{b}$ and $\eta_{u d}$ are calculated by setting $J_{d}$ and $\hat{\dot{m}}$, and then measuring $J_{s}, J_{b}$, and $V_{d}$. Performance curves are generated by incrementally decreasing $J_{d}$ from the nominal 30 A value and recording the new values. Unfortunately, increasing $J_{d}$ is not an option because the NEXT DCA has a maximum current rating of $30 \mathrm{~A}$.

The value of 0.35 in Equation 6 represents the fraction of ions that strike the ion collection grid and subsequently exit the discharge chamber. Previous researchers have found this value to be closer to 0.50 or $0.55 .{ }^{15,34}$ However, those results were obtained when utilizing the high-voltage ion optics as the ion collection grid. For the experiments presented here high-voltage ion optics are not utilized as the ion collection grid. Instead a grid with the same open area fraction, but larger diameter holes is utilized. In this case, if an exiting ion fraction of 0.50 is utilized, the propellant utilization efficiency is calculated to be greater than 1, an obviously anomalous result. An exiting ion fraction of 0.35 is chosen because this value ensures that all calculated propellant utilization efficiencies are less than 1 .

Beam flatness is calculated as the average beam current density divided by the peak beam current density. ${ }^{40}$ In this case the current density is measured at discrete points at the grid plane by the 13 grid-plane button probes. Flatness is calculated by averaging the current density of the 13 probes and then dividing by the maximum.

\section{Results and Analysis}

A stable discharge is successfully obtained for all magnetic field configurations investigated. Unfortunately, to operate stably, the -5 A electromagnet configuration requires an $8 \%$ larger DCA flow rate than the other configurations. Even during stable operation at the elevated flow rate, the -5 A electromagnet configuration has ion production costs and propellant utilization efficiencies that are $\sim 125 \%$ larger and $\sim 38 \%$ less, respectively, than any of the other configurations. As a result, the -5 A configuration is not further investigated or discussed.

The asymmetric magnetic field configuration is investigated to determine if increasing the magnetic field on the near-DCA side of the TA causes the grid-plane current peak location to change. Results indicate that an asymmetric magnetic field configuration does not cause the peak location to move.

Magnetic field configuration has a profound impact on TA operation. General results for all configurations as well as comparison of the TA with previous and current ion thruster discharge chambers is discussed in the following sections. Backplate current ratio, grid-plane plasma properties, and erosion considerations are discussed. Finally, the optimized TA magnetic field is determined based on the results and analysis presented. 


\section{A. Performance}

Characteristic performance curves for the TA are shown in Figure 5. TA performance curves show an increase in ion production cost for decreasing propellant utilization efficiency. This same trend has been documented by Sovey ${ }^{18}$ and Patterson ${ }^{41}$ for a $30 \mathrm{~cm}$ ring-cusp ion thruster and it has also been reported for the GRC HiPEP ion thruster. ${ }^{3}$ However, this result is uncommon and uncharacteristic of most ion thrusters, which typically show an increase in ion production cost as propellant utilization increases. ${ }^{15,18,34}$ This difference may be explained by the fact that the TA is a rectangular discharge chamber utilizing an entirely rectangular, ring-cusp magnetic field, where as most previous ion thrusters are cylindrical using a circular, ring-cusp magnetic field. Another explanation may be that discharge currents larger than $30 \mathrm{~A}$ need to be investigated. Because the NEXT DCA is limited to $30 \mathrm{~A}$, larger discharge currents, which may reveal a missing part of the performance curves, are not investigated. Decreasing the discharge current below 25 A may also show a change in the performance curve trends.

Magnetic field configuration has a significant effect on discharge chamber performance. As the backplate magnetic field is increased with the electromagnet, the grid-plane ion current increases. This trend has also been documented by Sovey ${ }^{18}$ and Patterson ${ }^{41}$ for a $30 \mathrm{~cm}$ ring-cusp discharge chamber. The +10 A electromagnet configuration provides the lowest production costs with the highest propellant utilizations, followed by the $+5 \mathrm{~A}$ and $0 \mathrm{~A}$ configurations, respectively. Although the $50 \mathrm{G}$ enclosed configuration increases the magnetic field along the anode walls of the TA, this does not decrease production costs.

Figure 6 shows ion production cost and propellant utilization efficiency as a function of magnetic field configuration for the nominal $30 \mathrm{~A}$ discharge with the center DCA active and no flow through the DCUs. Propellant efficiency increases with backplate magnetic field strength, while ion production costs decrease. Compared with contemporary discharge chambers, ion production costs for magnetic field configurations number 1 and 2 (50 G enclosed and electromagnet at $-10 \mathrm{~A}$, respectively) are slightly large, but the other magnetic field configurations obtain values that are reasonable. Performance numbers for other ion thruster discharge chambers can be found in Table 2.

\section{B. Uniformity and Flatness}

Magnetic field configuration has a significant impact on the uniformity of the grid-plane ion current. As the backplate magnetic field increases, the measured flatness increases and then subsequently decreases, reaching a value of 0.55 for the 0 A electromagnet configuration with the center DCA active. This trend is illustrated in Figure 6. Sovey's investigation found that increasing the upstream magnetic field reduced the flatness. ${ }^{18}$ This

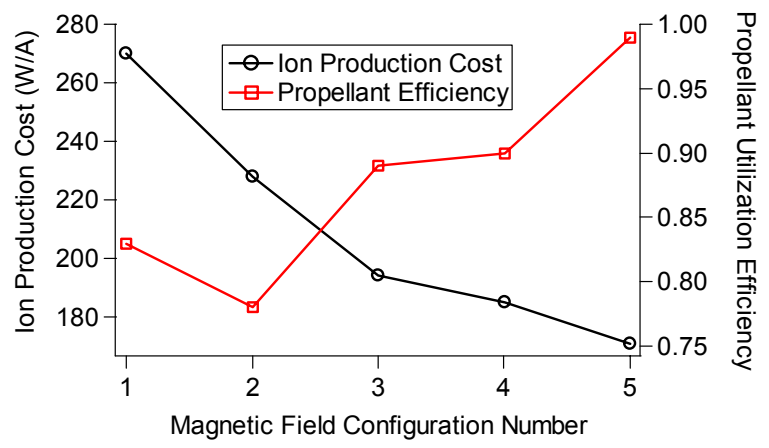

a.)

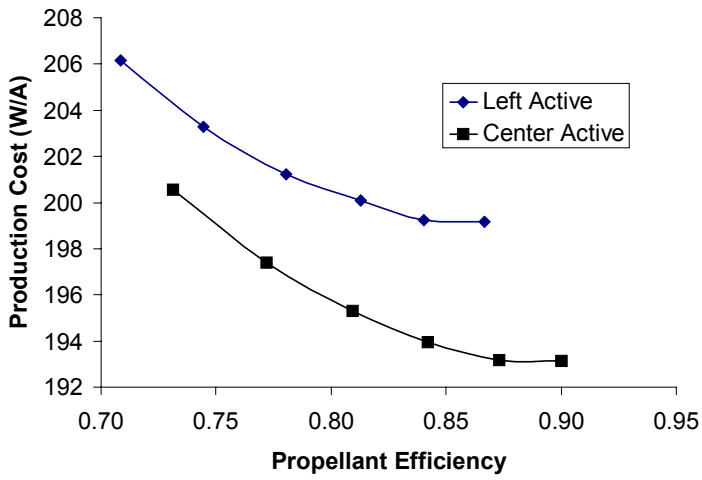

Figure 5: 0 A electromagnet configuration performance for both left and center DCA active configurations

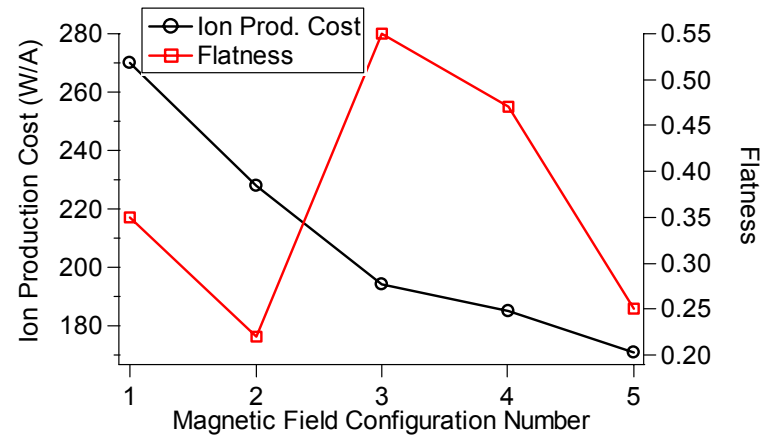

b.)

$1=50 \mathrm{G}$ enclosed, $2=-10 \mathrm{~A}, 3=0 \mathrm{~A}, 4=+5 \mathrm{~A}, 5=+10 \mathrm{~A}$

Figure 6: a.) Ion production cost and propellant utilization efficiency as a function of magnetic field configuration. b.) Ion production cost and flatness as a function of magnetic field configuration. Both are for a $30 \mathrm{~A}$ discharge with the center DCA active and no DCU flow.

10

American Institute of Aeronautics and Astronautics 
trend is also exhibited by the TA results, which show that increasing the upstream magnetic field too much can cause the flatness to decrease. Overall, these results suggest that when designing the magnetic field of a rectangular discharge chamber there is a trade-off between ion production cost, propellant utilization efficiency, and flatness.

Compared with contemporary discharge chambers, flatness for all magnetic field configurations except number 3 and 4 (electromagnet at $0 \mathrm{~A}$ and electromagnet at $+5 \mathrm{~A}$ respectively) is low. A comparison of TA flatness at the $0 \mathrm{~A}$ electromagnet configuration with other contemporary ion thruster discharge chambers is shown in Table 2 .

\begin{tabular}{|cccccc|}
\hline Thruster & Geometry & $\begin{array}{c}\text { Propellant } \\
\text { Efficiency }\end{array}$ & $\begin{array}{c}\text { Ion Production Cost } \\
\text { (W/A) or (eV/ion) }\end{array}$ & Flatness & $\begin{array}{c}\text { Real or Simulated } \\
\text { Beam Current (A) }\end{array}$ \\
\hline NSTAR $^{42,43}$ & Cylindrical & 0.89 & 173 & 0.47 & 1.76 \\
NEXT $^{40,44}$ & Cylindrical & 0.89 & 135 & 0.66 & 3.52 \\
NEXT $^{40,44}$ & Cylindrical & 0.90 & 205 & 0.57 & 1.20 \\
\hline NEXIS $^{34}$ & Cylindrical & 0.90 & 160 & 0.85 & 3.90 \\
HiPEP $^{3}$ & Rectangular & 0.90 & 188 & & \\
MCDC TA & Rectangular & 0.89 & 194 & 0.55 & 3.78 \\
\hline
\end{tabular}

Table 2: Comparison of contemporary ion thruster discharge chambers with the MCDC TA operated with the nominal 0 A electromagnet configuration.

\section{DCU Connectivity and Flow Rate}

The DCUs are operated either electrically connected or isolated from the electrical circuit while their flow rate is set at zero, half the DCA flow, and the full DCA flow rate. Total flow rate is kept constant during this procedure and discharge voltage is found to vary a maximum of $1 \mathrm{~V}$ as DCU flow increases. Very little performance change is seen with DCU flow rate and electrical connectivity adjustment. Flatness improves as DCU flow rate increases for all configurations except the $50 \mathrm{G}$ enclosed configuration. However, most improvements are only on the order of $2-3 \%$, with a maximum $10 \%$ increase for the 0 A configuration with the left DCA operational.

Figure 7 illustrates the effects of flow rate on the DCU keeper floating voltage and collected current. When the DCUs are isolated from the TA electrical circuit, an increase in DCU flow rate causes the floating voltages of both the cathode and keeper to increase. When electrically connected to the TA, the DCU cathode and keepers collect current. DCU keeper currents are similar to the active DCA keeper current, while DCU cathode currents are orders of magnitude less than the DCA cathode current. This is to be expected since the active DCA cathode is emitting the bulk of the discharge current through electron and ion production while the DCUs are simply collecting a current. As DCU flow rate increases, DCU keeper current decreases, but cathode current does not

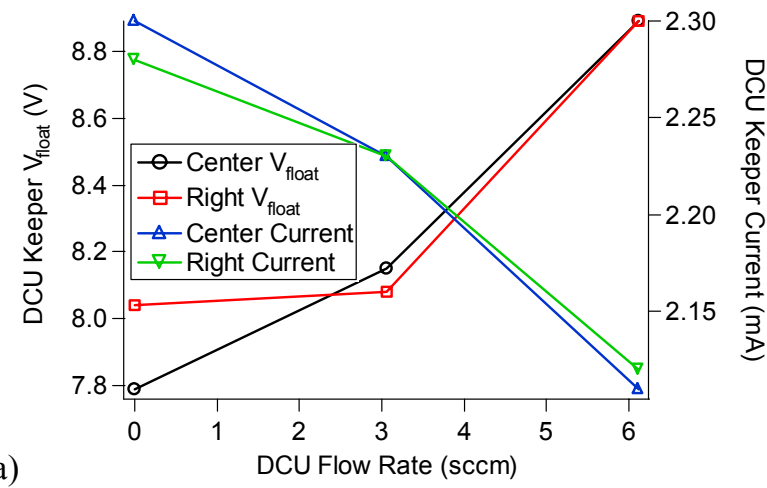

display a consistent trend. A decrease in keeper current with DCU flow rate signifies a decrease in plasma bombardment of the keeper, which may lead to a decrease in keeper erosion. 


\section{Backplate Electron Current Deposition}

Because the magnetic circuit of the MCDC utilizes rectangular magnet rings, the electron deposition to the intercusp corners is characterized. Two button probes are mounted to the backplate of the anode on the left side of the left DCA. One probe is placed at the corner of the rectangular TA, while the other is placed at the midplane. This allows the intercusp corner electron deposition to be compared with that obtained at the midplane. The results presented here utilize the ratio of the currents (corner probe current divided by mid-plane probe current).

Figure 8 shows typical trends associated with the backplate current ratio. Corner probe current is always larger than mid-plane probe current, which suggests that more of the discharge current is deposited into the corners of the rectangular TA than in the middle. On average, the backplate current ratio is approximately 10 , with a maximum of 24 and a minimum of 4 . DCU connectivity does not affect backplate current ratio; however, DCU flow rate does. For the $+5 \mathrm{~A}$ electromagnet configuration, the backplate current ratio decreases from approximately 21 to 4.5 as the DCU flow rate is increased to its maximum value. This same trend is noticeable for the 0 A configuration as well. Figure 8 shows backplate current ratio decreases with increasing DCU flow rate and when the left DCA is operational. These results suggest that the internal TA plasma becomes more uniform as the DCU flow rate increases.

\section{E. Grid-plane Plasma Properties}

Figure 9 shows the average grid-plane plasma properties as a function of magnetic field configuration. Electron temperature is largest for the $50 \mathrm{G}$ enclosed and +10 A electromagnet configurations. The minimum temperature is obtained by the $+5 \mathrm{~A}$ configuration, which yields a value of $3.5 \mathrm{eV}$.

Ion number density is lowest for the 0 A configuration and increases with increasing electromagnet current. This is expected because, as described above, an increase in the backplate magnetic field causes the grid-plane current to increase. The number density for the -10 A configuration is not presented because anomalous values for floating potential and plasma potential are obtained, making the calculations unreliable. Typical values for plasma potential and floating potential at the grid-plane are $27 \mathrm{~V}$ and $5 \mathrm{~V}$, respectively. Finally, electron number density is similar to the ion number density, a result consistent with quasi-neutral plasma.

\section{F. Erosion Issues}

Each of the two DCUs is constructed of a copper cathode tube with a chamfered orifice and a copper keeper tube with an orifice. Pre and post-test photographs of the DCU cathodes are shown in Figure 10. TA operation for the experiments presented here totaled approximately 50 hours. A post-test visual inspection of the DCUs shows changes in the cathode orifice. DCU keeper orifices do not show any changes or wear. The cathode orifice is no longer smooth and circular, but appears to have jagged edges around the inner diameter of the orifice. Changes associated with the left and center DCU (this DCU is on the left during center DCA operation and in the center during left DCA operation) are more pronounced than those for the right DCU.

Visually the DCUs appear to have material deposition in the cathode orifice, a result that is similar to the neutralizer cathode deposition that occurred during the extended life test at JPL. ${ }^{11,12}$ Pre and post-test measurements of the cathode orifice diameter reveals a decrease in diameter of approximately $10 \%$. The deposition to the DCU cathodes may be caused by back-sputtered ion collection grid material. Specifically, back-sputtering of grid material 
may have occurred during initial TA operation because the DCA flow rate was mistakenly set at an increased level. The increased flow rate effectively created a collimated plasma jet, which bombarded the collection grid and may have caused back-sputtering of grid material. However, no noticeable change is noticed on the upstream surface of the collection grid. It is also important to note that subsequent TA experiments with the correct flow rate have not shown any back-sputtering material deposition. It is unclear if these results can be attributed to DCU propellant flow or DCU electrical connectivity.

The TA operation results suggest it may be possible to reduce dormant cathode keeper erosion by keeping propellant flowing through them. Propellant flow reduces the collected keeper current, which suggests fewer ions are bombarding the keeper. Furthermore, propellant flow causes the keeper floating potential to increase. Kolasinski has shown that higher keeper potentials reduce keeper erosion. ${ }^{45}$ Ions bombarding the keeper are typically falling from the plasma potential to the keeper potential. For plasma potentials of $27 \mathrm{~V}$ this yields energies within the range of $17-23 \mathrm{~V}$.

The decrease in keeper current during propellant flow may be explained by charge-exchange (CEX) collisions. In a CEX collision, a "fast" ion interacts with a "slow" neutral, the product of which is a "fast" neutral and a "slow" ion. External propellant flow is known to cause CEX collisions and affect energy distributions near hollow cathodes. ${ }^{46}$ Flow through the DCUs may cause bombarding ions to suffer CEX collisions, the result of which could be a "fast" neutral moving toward the DCU and a "slow" ion created at a potential lower than the plasma potential such that its bombarding energy is significantly less than an ion falling through the full plasma to keeper potential. More information regarding plasma properties and detailed analysis of potential erosion phenomenon for the PEPL MCDC TA dormant cathodes can be found in Ref. 47.

\section{G. Optimized TA Magnetic Field Configuration}

Overall, the 0 A electromagnet current TA configuration provides the best performance. Flatness and performance are both considered to determine the optimum configuration. The 0 A configuration has a relatively high flatness, 0.55 and 0.53 , and relatively low discharge losses, 194 W/A at 0.89 propellant utilization and $199 \mathrm{~W} / \mathrm{A}$ at 0.87 propellant efficiency, for both center and left DCA operation, respectively. Other configurations have lower production costs, but they also have decreased flatness. The optimum configuration does not enclose the $50 \mathrm{G}$ line within the TA. For the nominal $30 \mathrm{~A}$ discharge current, the discharge voltage is $24.5 \mathrm{~V}$ with peak-to-peak oscillations less than $1 \mathrm{~V}$ and a simulated beam current of $3.78 \mathrm{~A}$.

\section{Summary and Conclusions}

A HiPEP-derivative discharge chamber is developed by the University of Michigan for operation with multiple discharge cathode assemblies (DCAs). The multiple cathode approach attempts to increase thruster lifetime by operating three DCAs sequentially, possibly providing a threefold increase in discharge life. A multiple-cathode discharge chamber (MCDC) test article (TA) is fabricated to determine the viability of the multiple-cathode approach. Multiple design iterations yield a rectangular, ring-cusp magnetic field design with the baseline magnetic field created with only permanent magnets. An electromagnet and magnetic iron c-channels are utilized to augment the magnetic field and a total of 7 magnetic field configurations are investigated.

Experimental testing of the TA is conducted in the LVTF at operating pressures on the order of $4 \times 10^{-6}$ Torr and the TA is operated as a simulated ion thruster. ${ }^{15}$ A NEXT DCA is used and two dormant cathode units (DCUs) are also mounted in the TA. Magnetic field configuration, DCU electrical connectivity, and DCU flow rate are adjusted while monitoring performance, uniformity, DCU floating voltages, DCU currents, backplate electron current ratio, and grid-plane plasma properties.

Both centerline (center) and off-centerline (left) DCA operation within the TA is obtained. Simulated performance curves and calculated flatness parameters show the optimal TA configuration performs within the same 
operational regimes as contemporary ion thruster discharge chambers with production cost, propellant utilization, and flatness values on the order of $194 \mathrm{~W} / \mathrm{A}, 0.89$, and 0.55 , respectively. DCU connectivity and flow rate do not affect TA performance. As DCU flow rate increases, DCU floating voltages increase and DCU keeper currents decrease, suggesting that DCU flow may shield the keeper and reduce keeper erosion. Because the TA is a rectangular discharge chamber, the ratio of corner to mid-plane anode electron deposition is monitored. Calculated backplate current ratios are on average 10, suggesting that considerably more discharge current is deposited in the corners of the rectangular TA than at the mid-plane. This ratio decreases with increasing DCU flow rate. Post-test inspections of the DCUs show material deposition in the cathode orifice. This result is most likely due to backsputtered ion collection grid material caused by an initial DCA flow rate error. The optimum magnetic field configuration is determined to be the 0 A electromagnet configuration. This configuration places the cathode exitplane at approximately $65 \mathrm{G}$ and does not enclose the $50 \mathrm{G}$ line. While other configurations have lower losses, the 0 A configuration balances both performance and flatness.

Any potential dormant cathode erosion phenomenon in an MCDC may be mitigated by operating those units with propellant flow. Propellant flow reduces the collected keeper current, which suggests fewer bombarding ions are present. Furthermore, propellant flow also increases the keeper floating potential. Kolasinski has shown that increased keeper potentials reduce erosion. ${ }^{45}$ Although these initial suggestions for mitigating any potential dormant cathode erosion are insightful, further investigation into the effect an operating cathode has on the two dormant units is required.

\section{Future Work}

Further testing will involve mapping the plasma inside the MCDC using a single Langmuir probe mounted to the PEPL High-speed Axial Reciprocating Probe (HARP) positioning system. The HARP has had extensive use at PEPL and has been used to characterize the interior plasma of both Hall thrusters ${ }^{48}$ and ion thrusters. ${ }^{49}$ Specifically the near-DCA and near-DCU plasma must be characterized in order to understand better any potential erosion phenomena that may be present and to determine the optimum operational configuration of the dormant cathodes. (Electrically connected or isolated with propellant flow or no flow) Based on these results, specifically the plasma potential measurements, single-particle ion trajectories will be calculated to determine the initial positions and velocities of ions impacting the dormant cathodes and active DCA. These simulations will also be attempted including doubly-charged ions to determine if they contribute to dormant cathode erosion.

Another focus will be analyzing the effect a single operating cathode has on the dormant units. The life test of the SPT-100 showed that an operating cathode can cause significant erosion of the non-operating cathode, thus reducing its lifetime.$^{50}$ PEPL will replace the two DCUs with diagnostic cylinders equal in size and composition to the active DCA. Initially, the DCU keepers will be outfitted with planar Langmuir probes to determine plasma properties at the DCU keeper orifice plate. Further testing will acquire axial profiles of plasma properties downstream and internal to the DCUs. Finally, a miniature retarding potential analyzer (RPA) will be placed inside the cathode of a DCU to obtain the energies of ions that may impact the cathode.

Also, the TA plasma will be modeled utilizing the University of California at Berkeley plasma code XOOPIC. ${ }^{51}$ Data output by the code will be compared with experimentally measured plasma properties from this work, as well as from the HARP mapping described above. Results will provide insight into the operation of a MCDC and whether dormant cathode erosion is a life-limiting concern. All together, these measurements and models will provide information on the severity of ion deposition onto the dormant cathodes and the limiting effect it may have on their lifetime.

\section{Acknowledgements}

We would like to thank the entire research group at PEPL who have been instrumental in this investigation, Dr. Daniel Herman for his assistance with the NEXT discharge cathode and the initial operation of the TA, Mr. Bailo Ngom for assistance with hardware construction, and Mr. Terry Larrow for fabricating the hardware used in this study. We would also like to thank Mr. Michael Patterson of NASA GRC for the financial support of this research through research grant NNC04GA67G and for use of government equipment. We would like to acknowledge Dr. John Foster (grant monitor) who has been the principal contact at NASA GRC.

\section{References}

${ }^{1}$ Dunning, J. W., Hamley, J., Jankovsky, R., Oleson, S., "An Overview of Electric Propulsion Activies at NASA," AIAA2004-3328, 40th Joint Propulsion Conference, Fort Lauderdale, FL, July 11-14, 2004. 
${ }^{2}$ Elliott, F., Foster, J. E., Patterson, M. J., "An Overview of the High Power Electric Propulsion (HiPEP) Project," AIAA2004-3453, 40th Joint Propulsion Conference, Fort Lauderdale, FL, July 11-14, 2004.

${ }^{3}$ Foster, J. E., Haag, T. W., Kamhawi, H., Patterson, M. J., Malone, S., Elliott, F., Williams, G. J., Sovey, J. S., Carpenter, C., "The High Power Electric Propulsion Thruster," AIAA-2004-3812, 40th Joint Propulsion Conference, Fort Lauderdale, FL, July 11-14, 2004.

${ }^{4}$ Patterson, M. J., Roman, R. F., Foster, J. E., "Ion Engine Development for Interstellar Precursor Missions," AIAA-20003811, 36th Joint Propulsion Conference, Huntsville, AL, July 16-19, 2000.

${ }^{5}$ Oleson, S., "Electric Propulsion Technology Development for the Jupiter Icy Moon Orbiter Project," AIAA-2004-3449, 40th Joint Propulsion Conference, Fort Lauderdale, FL., July 11-14, 2004.

${ }^{6}$ Rawlin, V. K., Williams, G. J., Pinero, L., Roman, R. F., "Status of Ion Engine Development for High Power, High Specific Impulse Missions," IEPC-01-096, 27th International Electric Propulsion Conference, Pasadena, CA, October 15-19, 2001.

${ }^{7}$ Soulas, G. C., Foster, J. E., Patterson, M. J., "Performance of Titanium Optics on a NASA $30 \mathrm{~cm}$ Ion thruster," AIAA-20003814, 36th Joint Propulsion Conference, Huntsville, AL, July 16-19, 2000.

${ }^{8}$ Haag, T. W., Patterson, M. J., Rawlin, V. K., Soulas, G. C., "Carbon-based Ion Optics Development at NASA GRC," IEPC01-94, 27th International Electric Propulsion Conference, Pasadena, CA, October 15-19, 2001.

${ }^{9}$ Soulas, G. C., "Improving the Total Impulse capability of the NSTAR Ion thruster with Thick-Accelerator-Grid Ion Optics," IEPC-01-081, 27th International Electric Propulsion Conference, Pasadena, CA, October 15-19, 2001.

${ }^{10}$ Foster, J. E., Roman, R. F., Soulas, G. C., Patterson, M. J., "Electron Backstreaming Mitigation via a Magnetic Grid," IEPC-01-91, 27th International Electric Propulsion Conference, Pasadena, CA, October 15-19, 2001.

${ }^{11}$ Sengupta, A., Brophy, J. R., Anderson, J. R., Garner, C. E., de Groh, K., Karniotis, T., Banks, B., "An Overview of the Results from the 30,000 Hr Life Test of Deep Space 1 Flight Spare Ion Engine," AIAA-2004-3608, 40th Joint Propulsion Conference, Fort Lauderdale, FL, July 11-14, 2004.

${ }^{12}$ Sengupta, A., Brophy, J. R., Goodfellow, K., "Status of the Extended Life Test of the Deep Space 1 flight spare engine after 30,352 hours of operation," AIAA-2003-4558, 39th Joint Propulsion Conference, Huntsville, AL, July 20-23, 2003.

${ }^{13}$ Kamhawi, H., Soulas, G. C., Patterson, M. J., Frandina, M. M., "NEXT Ion Engine 2000 Hr Wear Test Plume and Erosion Results," AIAA-2004-3792, 40th Joint Propulsion Conference, Fort Lauderdale, FL, July 11-14, 2004.

${ }^{14}$ Domonkos, M. T., Foster, C., Soulas, G. C., "Wear Testing and Analysis of Ion Engine Discharge Cathode Keeper," Journal of Propulsion and Power, Vol. 21, No. 1, pp. 102-110, Jan.-Feb. 2005.

${ }^{15}$ Brophy, J. R., "Simulated Ion Thruster Operation Without Beam Extraction," AIAA-90-2655, 21st International Electric Propulsion Conference, Orlando, FL, July 18-20, 1990.

${ }^{16}$ Chen, F. F., Introduction to Plasma Physics and Controlled Fusion, Vol. 1: Plasma Physics, Plenum Press, New York, 1984.

${ }^{17}$ Nicholson, D. R., Introduction to Plasma Theory, John Wiley \& Sons, Inc., New York, 1983.

${ }^{18}$ Sovey, J. S., "Improved Ion Containment using a Ring-Cusp Ion thruster," Journal of Spacecraft and Rockets, Vol. 21, No. 5, pp. 488-495, Sept. - Oct. 1984.

${ }^{19}$ Hershkowitz, N., Leung, K. N., Romesser, T., "Plasma Leakage through a Low-Beta Line Cusp," Physical Review Letters, Vol. 35, pp. 277-280, August 1975.

${ }^{20}$ Leung, K. N., Hershkowitz, N., MacKenzie, K. R., "Plasma Confinement by Localized Cusps," Physics of Fluids, Vol. 19, No. 7, pp. 1045-1053, July 1976.

${ }^{21}$ Knorr, G., Merlino, R. L., "The Role of Fast Electrons for the Confinement of Plasma by Magnetic Cusps," Plasma Physics and Controlled Fusion, Vol. 26, No. 2, pp. 433-442, 1984.

${ }^{22}$ Horiike, H., Akiba, M., Ohara, Y., Okumura, Y., Tanaka, S., "Cusp Width and Power Flow study at a High Power Magnetic Multipole Ion Source," Physics of Fluids, Vol. 30, No. 10, pp. 3268-3275, Oct. 1987.

${ }^{23}$ Foster, J. E., "Intercusp Electron Transport in an NSTAR-derivative Ion thruster," Journal of Propulsion and Power, Vol. 18, No. 1, pp. 213-217, January 2002.

${ }^{24}$ Dushman, S., Scientific Foundations of Vacuum Technique, Vol. 4, Wiley, New York, 1958.

${ }^{25}$ Walker, M. L. R., Gallimore, A. D., Chunpei, C., Boyd, I. D., "Pressure Map of a Facility as a function of Flow Rate to study Facility Effects," AIAA-2002-3815, 38th Joint Propulsion Conference, Indianapolis, IN, July 7-10, 2002.

${ }^{26}$ Stuart, R. V., Wehner, G. K., "Sputtering Yields at Very Low Bombarding Ion Energies," Journal of Applied Physics, Vol. 33, No. 7, pp. 2345-2352, July 1962.

${ }^{27}$ Rosenberg, D., Wehner, G. K., "Sputtering Yields for Low Energy $\mathrm{He}^{+}-, \mathrm{Kr}^{+}$-, and $\mathrm{Xe}^{+}$-Ion Bombardment," Journal of Applied Physics, Vol. 33, No. 5, pp. 1842-1845, May 1962.

${ }^{28}$ Foster, J. E., Personal Communication, NASA Glenn Research Center, Cleveland, OH., October, 2004.

${ }^{29}$ Reader, P. D., "Experimental Effects of Propellant Introduction Mode on Electron-Bombardment Ion Rocket Performance," NASA TN D-2587, 1965.

${ }^{30}$ Beattie, J. R., Matossian, J. N., "Mercury Ion Thruster Technology," NASA CR-174974, Hughes Research Laboratories, March 1989.

${ }^{31}$ Zikeyev, M. V., Shagayda, A. A., "Probe Measurements in Discharge Chamber of Low-Power Ion thruster," IEPC-03-120, 28th International Electric Propulsion Conference, Toulouse, France, March 17-21, 2003.

${ }^{32}$ Beattie, J. R., "Cusped Magnetic Field Mercury Ion thruster," Doctoral Thesis, Dept. of Mechanical Engineering, Colorado State University, Fort Collins, CO, 1976. 
${ }^{33}$ Wilbur, P. J., Isaacson, J., "Plasma Property Contours in a $15 \mathrm{~cm}$ Hollow Cathode Kaufman thruster," Dept. of Mechanical Engineering, Colorado State University, Fort Collins, CO, February 1973.

${ }^{34}$ Goebel, D. M., Polk, J. E., Sengupta, A., "Discharge Chamber Performance of the NEXIS Ion Thruster," AIAA-2004-3813, 40th Joint Propulsion Conference, Fort Lauderdale, FL, July 11-14, 2004.

${ }^{35}$ Chen, F. F., "Electric Probes," Plasma Diagnostic Techniques, R. H. Huddlestone and S. L. Leonard, eds., Academic Press, New York, 1965.

${ }^{36}$ Mott-Smith, H. M., Langmuir, I., "The Theory of Collectors in Gaseous Discharges," Physical Review, Vol. 28, pp. 727763, October 1926.

${ }^{37}$ Hershkowitz, N., "How Langmuir Probes Work," Plasma Diagnostics, O. Auciello and D. L. Flamm, eds., Academic Press, Inc., Boston, pp. 113-184,1989.

${ }^{38}$ Hutchinson, I. H., Principles of Plasma Diagnostics, Second Ed., Cambridge Universitiy Press, Cambridge, 2002.

${ }^{39}$ Domonkos, M. T., "Evaluation of Low-Current Orificed Hollow Cathodes," Doctoral Thesis, Dept. of Aerospace Engineering, University of Michigan, Ann Arbor, MI, 1999.

${ }^{40}$ Soulas, G. C., Haag, T. W., Patterson, M. J., "Performance Evaluation of 40cm Ion Optics for the NEXT Ion Engine," AIAA-2002-3834, 38th Joint Propulsion Conference, Indianapolis, IN, July 7-10, 2002.

${ }^{41}$ Patterson, M. J., "Performance Characteristics of Ring-Cusp Thrusters with Xenon Propellant," AIAA-86-1392, 22nd Joint Propulsion Conference, Huntsville, AL, June 16-18, 1986.

${ }^{42}$ Soulas, G. C., "Performance Evaluation of Titanium Ion Optics for the NASA $30 \mathrm{~cm}$ Ion thruster," IEPC-01-092, 27th International Electric Propulsion Conference, Pasadena, CA, October 15-19, 2001.

${ }^{43}$ Polk, J. E., Anderson, J. R., Brophy, J. R., Rawlin, V. K., Patterson, M. J., Sovey, J. S., Hamley, J., "An Overview of the Results from an 8200 Hour Wear Test of the NSTAR Ion thruster," AIAA-99-2446, 35th Joint Propulsion Conference, Los Angeles, California, June 20-24, 1999.

${ }^{44}$ Soulas, G. C., Kamhawi, H., Patterson, M. J., Britton, M. A., Frandina, M. M., "NEXT Ion Engine 2000 Hr Wear Test Results," AIAA-2004-3791, 40th Joint Propulsion Conference, Fort Lauderdale, FL, July 11-14, 2004.

${ }^{45}$ Kolasinski, R. D., Polk, J. E., "Characterization of Cathode Keeper Wear by Surface Layer Activation," Journal of Propulsion and Power, Vol. 20, No. 6, pp. 992-999, Nov.-Dec. 2004.

${ }^{46}$ Kameyama, I., Wilbur, P. J., "Effects of External Flow near High-Current Hollow Cathodes on Ion-Energy Distributions," IEPC-97-173, 25th International Electric Propulsion Conference, Cleveland, OH, Aug. 24-28, 1997.

${ }^{47}$ Rovey, J. L., Gallimore, A. D., "Dormant Cathode Plasma Properties and Erosion Analysis in a Multiple-Cathode, HighPower, Rectangular Discharge Chamber," AIAA-2005-4241, 41st Joint Propulsion Conference, Tucson, AZ, July 10-13, 2005.

${ }^{48}$ Haas, J. M., Gallimore, A. D., "Characterization of the Internal Plasma Structure of a 5kW Hall thruster," IEPC 99-078, 26th International Electric Propulsion Conference, Kitakyushu, Japan, October 1999.

${ }^{49}$ Herman, D. A., McFarlane, D. S., Gallimore, A. D., "Discharge Plasma Parameters of a $30 \mathrm{~cm}$ Ion thruster measured Without Beam Extraction using a High-speed Axial Reciprocating Probe positioning system," IEPC-03-0069, 28th International Electric Propulsion Conference, Toulouse, France, March 17-21, 2003.

${ }^{50}$ Garner, C. E., Brophy, J. R., Polk, J. E., Pless, L. C., "Cyclic Endurance Test of a SPT-100 Stationary Plasma thruster," AIAA-94-2856, 30th Joint Propulsion Conference, Indianapolis, IN, June 27-29, 1994.

${ }^{51}$ Verboncoeur, J. P., Langdon, A. B., Gladd, N. T., "An object-oriented electromagnetic PIC code," Computer Physics Communications, Vol. 87, pp. 199-211, May 1995. 